\title{
Thresholds for the formation of satellites in two-dimensional vortices
}

\author{
By M. R. TURNER AND A. D. GILBERT \\ Mathematics Research Institute, School of Engineering, Computing and Mathematics, \\ University of Exeter, Exeter EX4 4QF, U.K.
}

(Received 7 July 2008)

This paper examines the evolution of a two-dimensional vortex which initially consists of an axisymmetric monopole vortex with a perturbation of azimuthal wavenumber $m=2$ added to it. If the perturbation is weak then the vortex returns to an axisymmetric state and the non-zero Fourier harmonics generated by the perturbation decay to zero. However, if a finite perturbation threshold is exceeded, then a persistent nonlinear vortex structure is formed. This structure consists of a coherent vortex core with two satellites rotating around it.

The paper considers the formation of these satellites by taking an asymptotic limit in which a compact vortex is surrounded by a weak skirt of vorticity. The resulting equations match the behaviour of a normal mode riding on the vortex with the evolution of finescale vorticity in a critical layer inside the skirt. Three estimates of inviscid thresholds for the formation of satellites are computed and compared: two estimates use qualitative diagnostics, the appearance of an inflection point or neutral mode in the mean profile. The other is determined quantitatively by solving the normal mode/critical-layer equations numerically. These calculations are supported by simulations of the full Navier-Stokes equations using a family of profiles based on the tanh function.

\section{Introduction}

It has been observed in two-dimensional, high Reynolds number flows that axisymmetric, coherent vortical structures arise spontaneously from random initial conditions (McWilliams 1984; Fornberg 1977). Many studies of these coherent structures focus on the behaviour of an axisymmetric vortex under a non-axisymmetric perturbation, which could be weak or strong. These studies typically consider perturbations with an azimuthal wavenumber $m=2$ or higher. (For discussion of the $m=1$ case and its peculiarities see Llewellyn Smith 1995.) Early studies of such vortices showed that they often return to an axisymmetric state. A vortex can relax inviscidly to an axisymmetric state when the non-axisymmetric perturbation becomes finely scaled due to the spiral wind-up in the underlying flow (Melander et al. 1987). As the values of the perturbation vorticity do not tend to zero pointwise, a more convenient notion of axisymmetrization is when the non-axisymmetric components of the stream function $\psi$ tend to zero for large times. For large but finite Reynolds number $R$, the non-axisymmetric components of the vorticity are destroyed, and hence the vortex axisymmetrizes, on the time scale $O\left(R^{1 / 3}\right)$, which is fast compared to the slow viscous timescale of $O(R)$ (Bernoff \& Lingevitch 1994; Bajer et al. 2001).

As more numerical and experimental studies were carried out, it became apparent that axisymmetrization only occurs for sufficiently weak perturbation amplitudes, and 
that for larger amplitudes the vortex can evolve into a persistent non-axisymmetric state (Dritschel 1989, 1998; Koumoutsakos 1997; Rossi et al. 1997). An example of a persistent nonlinear state is a multipole, which can be formed by allowing an initial vorticity distribution of the form

$$
Z(r, \theta)=Z_{0}(r)+\delta Z_{m}(r) e^{i m \theta}+\text { complex conjugate, }
$$

to evolve freely in time. The variables $(r, \theta)$ are the usual polar coordinates and $Z_{m}(r) e^{i m \theta}$ is an $m$-fold perturbation to the axisymmetric monopole vortex $Z_{0}(r)$. The real parameter $\delta>0$ is an amplitude and axisymmetrization occurs if $\delta$ is below a threshold value. In the most studied case the vorticity distributions take the form

$$
Z_{0}(r)=\frac{1}{4 \pi} e^{-r^{2} / 4}, \quad Z_{2}(r)=\frac{r^{2}}{4 \pi} e^{-r^{2} / 4}
$$

with $m=2$. The vortex evolves into a tripole which consists of a vortex core with two opposite-signed satellites of vorticity rotating around it, if $\delta$ is above some threshold value (Rossi et al. 1997; Barba 2006; Barba \& Leonard 2007). This tripole structure can be seen in the top row of panels in figure 1, which show the evolution of (1.1) and (1.2) with $R=10^{4}$ and $\delta=0.5$ at $t=0, t=300$ and $t=700$. Negative vorticity is coloured black in the panels, and at $t=700$ we can see the two negative satellites rotating around a positive vortex core.

Such tripoles can also emerge from instabilities within axisymmetric shielded monopoles, that is comprising a vortex core with a ring of opposite-signed vorticity encasing it and zero total circulation. This has led to many investigations of the evolution of a tripole from these shielded monopoles by experiments (Van Heijst et al. 1991) and numerical studies (Carton \& Legras 1994; Morel \& Carton 1994). The physical observation of these structures is not restricted to the laboratory however, as these tripoles have been observed in the ocean, first by Pingree \& Le Cann (1992).

There remain a number of unresolved issues involving such multipolar vortices. For what choices of $Z_{0}(r)$ and $Z_{m}(r)$ do such structures form? How, if at all, are these multipoles (which have both signs of vorticity present) related to persistent cat's eye structures in which the vorticity in the satellites is the same sign as the core? The threshold parameter $\delta$ shows a dependence on the Reynolds number: does $\delta(R)$ have a limiting value in the inviscid limit as $R \rightarrow \infty$ ? Barba \& Leonard (2007) have run simulations showing that the threshold value $\delta(R)$ decreases as $R$ is increased, but the large Reynolds number limit has not been extensively researched.

The aim of this paper is to address some of these issues using a combination of numerical simulations and an asymptotic limit in which we consider vortices with sharp edges. To set the scene, we note that the phenomenon of tripole formation is not restricted to Gaussian vortices: consider, for example the 'tanh' profiles

$$
\begin{aligned}
& Z_{0}(r)=\frac{1}{4 \pi} \frac{1-\tanh \left(\left(r^{2}-\sigma^{2}\right) /(4(1-\sigma))\right)}{1+\tanh \left(\sigma^{2} /(4(1-\sigma))\right)} \\
& Z_{2}(r)=\frac{r^{2}}{4 \pi} \frac{1-\tanh \left(\left(r^{2}-\sigma_{2}^{2}\right) /\left(4\left(1-\sigma_{2}\right)\right)\right)}{1+\tanh \left(\sigma_{2}^{2} /\left(4\left(1-\sigma_{2}\right)\right)\right)}
\end{aligned}
$$

(Hall et al. 2003a). As $\sigma$ and $\sigma_{2}$ are varied from 0 to 1 , the axisymmetric vortex and the perturbation change from a broad Gaussian-like form to a sharp Rankine (top-hat) vortex. The bottom row of panels in figure 1 shows the evolution of these tanh profiles into a tripole for the parameter values $\sigma=\sigma_{2}=0.2, R=10^{4}$ and $\delta=0.5$. In this case the negative satellites are clearly visible in each panel as the black regions outside the 

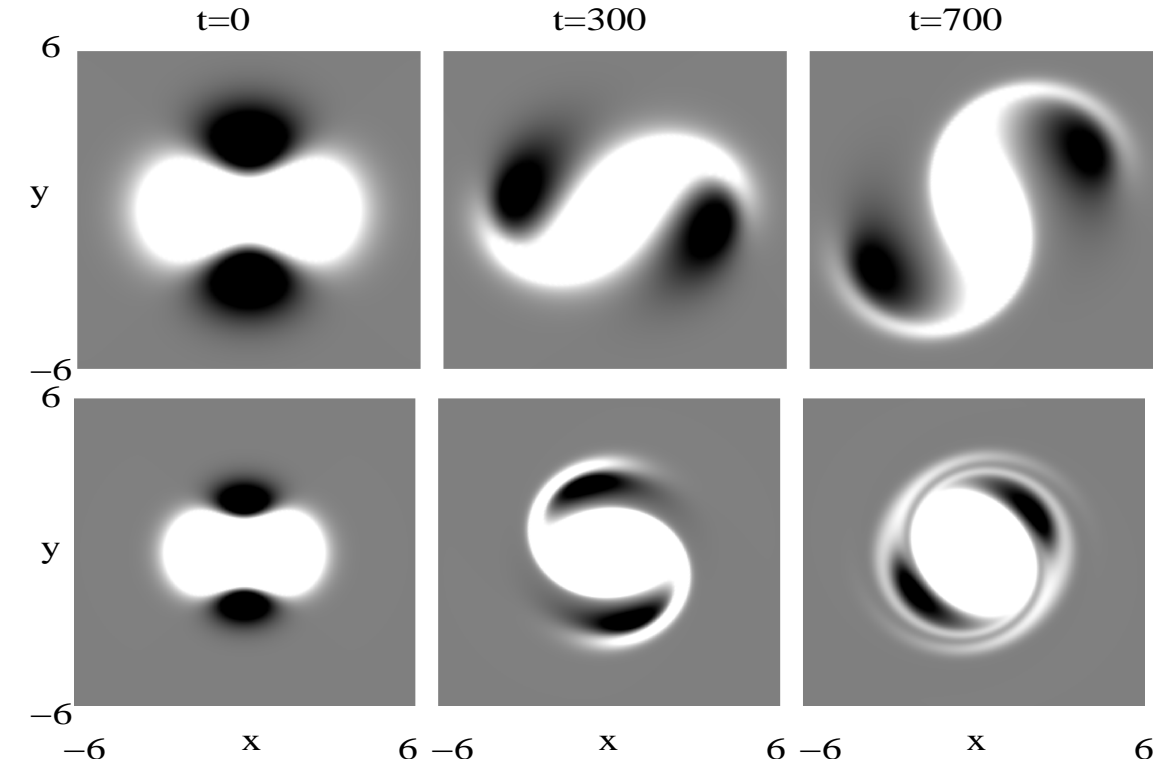

Figure 1. Plot of the Gaussian vortex (top row) and the tanh profile vortex (bottom row), with $\sigma=\sigma_{2}=0.2$, relaxing to a tripole structure with $R=10^{4}$ and $\delta=0.5$. Positive vorticity is shown as white, negative vorticity is black and zero vorticity is grey; the peak vorticity values are saturated at $|Z|=0.005$.

vortex core. Note that while such sharp edged vortices are convenient mathematically, they also arise very naturally in geophysical systems and two-dimensional turbulence via processes such as vortex stripping (Legras \& Dritschel 1993).

Using these tanh profiles in the limit as $\sigma \rightarrow 1$ we are able to calculate an inviscid threshold for the formation of satellite vortices using the theory of Balmforth et al. (2001), henceforth referred to as BLSY. As mentioned above, another type of non-axisymmetric structure that can form is a ring of cat's eyes around a vortex core, which has vorticity everywhere of one sign: here the fluid circulates about each cat's eye (in a frame corotating with the ring) and the vorticity is locally flattened. To be clear about our terminology, we will use the term cat's eyes to refer cases where the vorticity is everywhere of one sign, multipoles for both signs, and satellites to refer to either. These cat's eyes may be generated for $m=2$ by exposing an axisymmetric vortex to an irrotational external strain field that is switched off after some given time. For a sufficiently weak strain the vortex returns to an axisymmetric state, and the non-axisymmetric perturbations are wound up into spiral filaments (Briggs et al. 1970; Bassom \& Gilbert 1998; Le Dizès 2000). If the amplitude of the strain is above some threshold amplitude however, the vorticity can locally homogenize to form a pair of cat's eyes rotating around the coherent vortex core (Schecter et al. 2000; Balmforth et al. 2001; Macaskill et al. 2002; Turner \& Gilbert 2007). Thus for $m=2$, cat's eyes appear similar to the satellites in a tripole vortex, except they are of the same sign as the core.

Thresholds for cat's eye formation in a Gaussian vortex have been obtained numerically by Turner \& Gilbert (2007) and by BLSY in their asymptotic model, with qualitative agreement. In this paper our aim is to study the threshold for the formation of satellite structures for sharp-edged vortices using the asymptotic model of BLSY in the absence of an external forcing but with an initial condition equivalent to that in (1.1) used for the formation of multipolar vortices. This model begins with a smooth monopole vortex 
with the profile

$$
Z_{0}(r)=Z_{C}(r)+\epsilon Z_{S}(r)
$$

which consists of a compact vortex $Z_{C}(r)$, that is where the vorticity is identically zero beyond some radius, plus an asymptotically small 'skirt' $\epsilon Z_{S}(r)$. The small parameter that is exploited is $\epsilon$. The simplest example of a compact vortex is a Rankine vortex, where

$$
Z_{C}(r)= \begin{cases}1, & (r<1), \\ 0, & (r>1) .\end{cases}
$$

This vortex supports an $m=2$ Kelvin mode with a critical layer at $r_{2}=\sqrt{2}$ where the angular velocity of the mode equals that of the fluid. When a weak skirt is introduced this wave interacts with vorticity in the critical layer of width $\epsilon$ and the normal mode is replaced by a quasi-mode (Briggs et al. 1970; Schecter et al. 2000; Turner \& Gilbert 2007). A quasi-mode is essentially a special transient solution of the linear initial-value problem where the stream function decays exponentially and the vorticity wraps up into spiral filaments without decay.

The BLSY model yields a coupled system comprising an ODE for the normal mode amplitude and a PDE for the vorticity in the critical layer, and these authors study this system when the vortex is subjected to an external strain field. For weak strain there is the linear quasi-mode and exponential decay, but for stronger perturbations, above a threshold, persistent cat's eyes form, as vorticity is homogenized in recirculating regions in the critical layer. We also note in passing that rather than the BLSY approach of solving the initial value problem, another study (Le Dizès 2000) seeks steady states with asymptotically thin cat's eyes, using a jump condition across the critical layer.

We adapt the asymptotic theory of BLSY to incorporate a non-zero initial mode $m$ condition, such as the examples (1.1) to (1.4). This only involves two complex parameters, the initial amplitude of the normal mode and the initial strength of the mode $m$ vorticity in the critical layer, and these reduce to three real parameters after rotation. Thus the question of the formation of satellite vortices, within the limit of sharp-edged vortices (or similar ones fitting the BLSY framework) amounts to obtaining a threshold in a threedimensional parameter space, when the initial condition is allowed to evolve freely in time. Although we will be able to calculate a threshold for the formation of satellites, we will not be able to determine the sign of the vorticity in the satellites because the governing equations for the vorticity are identical to those where the vorticity has an arbitrary constant added to it. Focusing on the $m=2$ case, we study three methods of computing a threshold contour. The first two are only qualitative: an inflection point diagnostic calculates when the azimuthally averaged profile first develops an inflection point at the critical radius, and a neutral mode diagnostic calculates when this azimuthal averaged profile supports a neutral mode. We then simulate the BLSY system to obtain the true asymptotic threshold using a 'first bounce' criterion for when the vorticity perturbations feed back onto the basic profile strongly enough to produce satellites. We discuss the usefulness of the qualitative diagnostics and confirm the results by full Navier-Stokes simulations of profiles of the form (1.3) and (1.4).

The structure of the paper is as follows. In $\S 2$ we give the BLSY governing equations and discuss initial conditions. In $\S 3$ we take a weakly nonlinear version of the BLSY equations and use this to calculate both the inflection point and the neutral mode thresholds. Simulations of the BLSY system give the correct asymptotic threshold and are detailed in $\S 4$ while comparison with full Navier-Stokes simulations is given in $\S 5$. Some concluding remarks are set out in $\S 6$. 


\section{Formulation}

In this section we give the equations from the asymptotic theory of BLSY. We use their notation and we direct the reader to this paper for derivations, in particular rescalings, and discussion (though a few results we need are to be found in appendix B). The theory begins with a profile of the form (1.5) which supports a normal mode with complex amplitude $\hat{\varphi}(t)$. This interacts with the total vorticity $\zeta_{T}=\beta y+\zeta(y, \theta, t)$ in a thin critical layer, where $y$ is a rescaled, inward pointing radial coordinate with the critical layer at $y=0$. The parameter $\beta= \pm 1$ specifies the background gradient; our interest is only in the case $\beta=1$ so that the background profile is increasing with $y$, so decreasing with radius $r$, and the vortex is stable. The equations are, with $m=2$ from now on:

$$
\begin{gathered}
\partial_{t} \zeta+y \partial_{\theta} \zeta+\left(\beta+\partial_{y} \zeta\right) \partial_{\theta} \varphi=0, \\
i \partial_{t} \hat{\varphi}=\chi(t)+\left\langle e^{-2 i \theta} \zeta\right\rangle, \\
\varphi(\theta, t)=\hat{\varphi}(t) e^{2 i \theta}+\text { c.c. }, \\
\langle f\rangle=\mathrm{PV} \int_{-\infty}^{\infty} d y \oint \frac{f(y, \theta, t)}{2 \pi} d \theta .
\end{gathered}
$$

The normal mode of amplitude $\hat{\varphi}(t)$, riding on the compact vortex, drives a flow with stream function $\varphi(\theta, t)$ defined by $(2.3)$ in the critical layer, and (2.1) gives advection of vorticity in this and the background shear from the compact vortex. Equation (2.2) then gives the feedback on the normal mode with a principal value integral defined by (2.4).

We set the external forcing, that is strain, $\chi(t)$ to zero, but instead are interested in adding an initial mode 2 disturbance, as in (1.1)-(1.4). In limits for which the BLSY theory applies, any initial disturbance of this form amounts to specifying the initial complex amplitude $\Delta$ of the normal mode, and the initial complex value of the mode 2 vorticity in the critical layer, given by another complex constant $\Gamma$. We thus use the initial condition

$$
\zeta(y, \theta, t=0)=\Gamma_{0}+\Gamma e^{2 i \theta}+\text { c.c. }, \quad \hat{\varphi}(t=0)=\Delta .
$$

Here the additive constant $\Gamma_{0}$, the mean vorticity in the critical layer, does not affect the BLSY analysis as it does not enter into any of (2.1-2.4); hence we set $\Gamma_{0}=0$ throughout the analysis. However the value of this parameter in the full Navier-Stokes simulations ultimately determines if the satellites formed are positive (cat's eyes) or negative (a tripole). Since after rotation we can take one of $\Gamma$ or $\Delta$ to be purely real, we are left with a three-dimensional parameter space, and the question: for which points in this space do persistent structures form in the critical layer? By solving (2.1-2.5) in both weakly nonlinear and fully nonlinear settings we will calculate the inflection point, neutral mode and first bounce thresholds, above which the nonlinear satellites induced on the vortex persist and below which the vortex returns to an axisymmetric state. These thresholds will be of the form

$$
F(\Gamma, \Delta)=0,
$$

which gives a surface in parameter space. The results we will obtain are universal in that they apply to any vortex for which the BLSY model is valid.

However to compare with full Navier-Stokes simulations, we shall focus on the family of profiles in (1.3), which tend to the Rankine vortex (1.6) as $\sigma \rightarrow 1$. In this case the $m=2$ critical layer occurs at $r_{2}=\sqrt{2}$ and so the small parameter $\epsilon$ for the asymptotic expansions is proportional to $Z_{0}^{\prime}\left(r_{2}\right)$, tending to zero as $\sigma \rightarrow 1$. In this case the constants 
$\Gamma$ and $\Delta$ are related to the parameters $\delta$ and $\sigma_{2}$ from (1.4) via

$$
\Gamma=\frac{\delta Z_{2}\left(r_{2}\right)}{\pi Z_{0}^{\prime}\left(r_{2}\right)^{2}}, \quad \Delta=\frac{8 \delta P_{2}}{\pi Z_{0}^{\prime}\left(r_{2}\right)^{2}},
$$

where $P_{2}$ is a projection on the normal mode, given by

$$
P_{2}=-\frac{1}{8}\left(\int_{0}^{1} s^{3} Z_{2}(s) d s+\mathrm{PV} \int_{1}^{\infty} \frac{s Z_{2}(s)}{2-s^{2}} d s\right) .
$$

These rescalings and this projection formula are explained, in a general setting, in appendix B.

Because of the principal value integral in (2.4), the early evolution of this system with the given initial condition has a singular forcing of the normal mode (cf. BLSY section 5.1). To cleanly handle this behaviour we integrate over the short time interval from $t=0$ to a time $t^{*}$ satisfying $1 \gg t^{*} \gg 1 / L$, where $L$ is some large cut-off value of the principal value integral. We refer to this latter time $t^{*}$ as $t=0^{+}$for brevity. For very short times the $\left(\beta+\partial_{y} \zeta\right) \partial_{\theta} \varphi$ term in (2.1) is much smaller than the other two terms, and so we have

$$
\zeta(y, \theta, t) \simeq \Gamma e^{-2 i y t+2 i \theta}+\text { c.c. },
$$

and then

$$
i \partial_{t} \hat{\varphi}=\left\langle\zeta e^{-2 i \theta}\right\rangle \simeq \Gamma \frac{\sin (2 L t)}{t} .
$$

Therefore on the short time scale of $t=O\left(L^{-1}\right)$ a large forcing of the quantity $\hat{\varphi}$ takes place. Using

$$
\int_{0}^{\infty} \frac{\sin (u)}{u} d u=\frac{\pi}{2},
$$

we find that at time $t=0^{+}, \hat{\varphi}\left(0^{+}\right)=\Delta-i \pi \Gamma / 2$.

We rewrite the initial value problem (for numerical and analytical reasons), now starting at time $t=0^{+}$by writing

$$
\zeta^{\prime}=\zeta-\left(\Gamma e^{-2 i y t+2 i \theta}+\text { c.c. }\right),
$$

to obtain the system of equations

$$
\begin{gathered}
\partial_{t} \zeta+y \partial_{\theta} \zeta^{\prime}+\left(\beta+\partial_{y} \zeta^{\prime}-2 i t \Gamma e^{-2 i y t+2 i \theta}+2 i t \Gamma^{*} e^{2 i y t-2 i \theta}\right) \partial_{\theta} \varphi=0, \\
i \partial_{t} \hat{\varphi}=\left\langle e^{-2 i \theta} \zeta^{\prime}\right\rangle, \quad \varphi=\hat{\varphi}(t) e^{2 i \theta}+\text { c.c. }
\end{gathered}
$$

(where the star denotes the complex conjugate) with the new initial conditions

$$
\zeta^{\prime}\left(y, \theta, 0^{+}\right)=0, \quad \hat{\varphi}\left(0^{+}\right)=\Delta-i \pi \Gamma / 2 .
$$

In the next section we derive the weakly nonlinear diagnostics we use for calculating our threshold criteria.

\section{Weakly nonlinear results}

In this section we consider a weakly nonlinear solution to (2.13-2.15) and derive the inflection point and the neutral mode diagnostics for determining when satellites persist in a vortex or when the vortex returns to an axisymmetric state. 


\subsection{Weakly nonlinear critical-layer equations}

We seek an approximate solution to (2.13-2.15) by introducing

$$
\zeta^{\prime}(y, \theta, t)=\hat{\zeta}^{\prime}(y, t) e^{2 i \theta}+\text { c.c. }+\bar{\zeta}(y, t)+\cdots .
$$

This expansion neglects all the higher order effects; however it does allow us to calculate the two qualitative criteria described above which have been used in the study of BLSY. Substituting (3.1) into (2.13) we obtain the set of equations

$$
\begin{aligned}
\partial_{t} \hat{\zeta}^{\prime}+2 i y \hat{\zeta}^{\prime}+2 i \beta \hat{\varphi} & =0 \\
\partial_{t} \bar{\zeta}-2 i\left(\partial_{y} \hat{\zeta}^{\prime}-2 i t \Gamma e^{-2 i y t}\right) \hat{\varphi}^{*}+\text { c.c. } & =0 \\
i \partial_{t} \hat{\varphi} & =\left\langle\hat{\zeta}^{\prime}\right\rangle .
\end{aligned}
$$

The initial conditions for this problem are

$$
\hat{\zeta}^{\prime}\left(y, 0^{+}\right)=0, \quad \bar{\zeta}\left(y, 0^{+}\right)=0, \quad \text { and } \quad \hat{\varphi}\left(0^{+}\right)=\Delta-i \pi \Gamma / 2 .
$$

The quantity $\bar{\zeta}$ is the correction to the azimuthal mean of the vorticity in the critical layer and we seek the large-time form of this function to see how the initial condition eventually alters the vorticity profile in the skirt. From this modification we can form diagnostics to test whether satellites persist or not. These are only qualitative as the weakly nonlinear development does not apply quantitatively at finite values, that is not at the threshold.

We solve (3.2) by the same method as in $\S 5.1$ of BLSY, and using the initial conditions (3.5) gives

$$
\begin{aligned}
\hat{\varphi} & =\left(\Delta-\frac{i \pi \Gamma}{2}\right) e^{-\pi \beta t}, \\
\hat{\zeta}^{\prime} & =2 i \beta \frac{(\beta \pi+2 i y)}{\beta^{2} \pi^{2}+4 y^{2}}\left(e^{-\pi \beta t}-e^{-2 i y t}\right)\left(\Delta-\frac{i \pi \Gamma}{2}\right) .
\end{aligned}
$$

Thus the large time form of the solution for $\bar{\zeta}$ in (3.3) can be expressed as

$$
\bar{\zeta}^{\infty} \equiv \bar{\zeta}(y, t \rightarrow \infty)=-32 \beta y \frac{|A|^{2}+\pi\left(A_{i} \Gamma_{r}-A_{r} \Gamma_{i}\right)}{\left(\beta^{2} \pi^{2}+4 y^{2}\right)^{2}}+8\left(\beta^{2} \pi^{2}-4 y^{2}\right) \frac{A_{r} \Gamma_{r}+A_{i} \Gamma_{i}}{\left(\beta^{2} \pi^{2}+4 y^{2}\right)^{2}},
$$

where $A=\Delta-i \pi \Gamma / 2$ and the subscripts $r$ and $i$ denote real and imaginary parts respectively. As mentioned earlier, we can rotate our axes to make one of the quantities $\Gamma$ or $\Delta$ real; thus without loss of generality we set $\Gamma_{r}=\Gamma$ and $\Gamma_{i}=0$.

\subsection{Inflection point diagnostic}

Here we consider an inflection point diagnostic used by BLSY: we consider the contour in parameter space where the azimuthally averaged total vorticity in the critical layer $\zeta_{\mathrm{T}}=\beta y+\bar{\zeta}^{\infty}$ first develops an extremal point at the critical radius $(y=0)$. Using this diagnostic with $\bar{\zeta}^{\infty}$ given by (3.8) and with $\Gamma$ real gives the expression

$$
F(\Gamma, \Delta)=\Delta_{r}^{2}+\Delta_{i}^{2}-\frac{\pi^{2}}{4} \Gamma^{2}-\frac{\pi^{4}}{32}=0 .
$$

Equation (3.9) gives a three-dimensional surface in the parameter space $\left(\Gamma, \Delta_{r}, \Delta_{i}\right)$ outside which satellites persist and within which the vortex returns to axisymmetry (i.e. the stream function $\hat{\varphi}(t)$ decays to zero).

For the case when $\Delta$ is also real, which corresponds to an initial condition of the form 


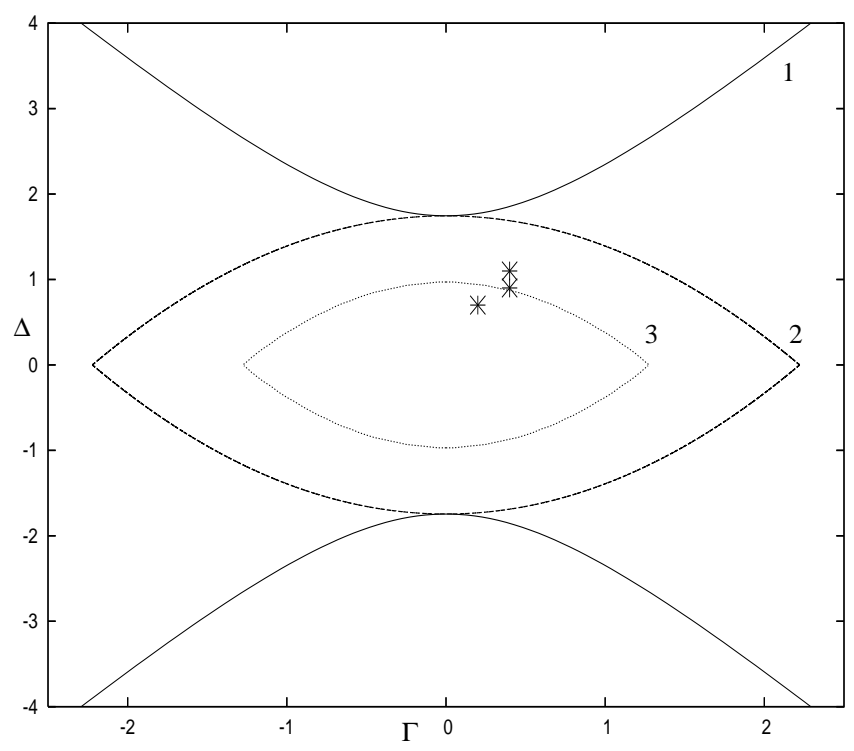

Figure 2. Threshold contours, for the case when $\Gamma$ and $\Delta$ are real, inside which satellites do not persist and outside which they do. Contour 1 is given by the inflection point diagnostic (3.10), contour 2 by the neutral mode diagnostic (3.16) and contour 3 by the numerical solution of (2.13-2.15). Contour 3 defines the set of parameters for which the first bounce time $t=45$. The stars represent parameter values used in the numerical simulations in $\S 4$.

used by the Navier-Stokes simulations of Rossi et al. (1997) and Barba \& Leonard (2007), we have a contour in the $(\Gamma, \Delta)$ plane given by

$$
F(\Gamma, \Delta)=\Delta^{2}-\frac{\pi^{2}}{4} \Gamma^{2}-\frac{\pi^{4}}{32}=0,
$$

which describes two hyperbolae centred on the point $\Delta=\Gamma=0$. For any parameter values lying between these hyperbolae the vortex would be assumed to return to an axisymmetric state and not contain persistent satellites. This contour can be seen as contour 1 in figure 2.

This inflection point threshold is very simplistic so we do not expect it to agree well with nonlinear simulations. This is because the existence of an extremal point does not guarantee satellite persistence, as was observed by BLSY for the generation of cat's eyes. Möller \& Montgomery (1999) have also used this method in the context of the full equations of motion and reached similar conclusions to the limitations of this diagnostic. A more informative diagnostic is to calculate when the vortex has an axisymmetric basic profile that will maintain a neutral mode, i.e., one where $\hat{\varphi}(t)=$ constant. Neutral modes of an axisymmetric vortex have been seen to correspond to persistent cat's eye structures (Turner et al. 2008).

\subsection{Neutral mode diagnostic}

To calculate neutral modes of the axisymmetric total vorticity profile in the critical layer $\zeta_{\mathrm{T}}=\beta y+\bar{\zeta}^{\infty}$, we perform a normal mode analysis. This analysis is the same as that in Hall et al. (2003a) and similar to that in BLSY. From (2.1) and (2.2) we solve the linearised system

$$
\partial_{t} \hat{\zeta}+i m y \hat{\zeta}+i m\left(\beta+\partial_{y} \bar{\zeta}^{\infty}\right) \hat{\varphi}=0, \quad i \partial_{t} \hat{\varphi}=\mathrm{PV} \int_{-\infty}^{\infty} \hat{\zeta}(y, t) d y
$$


where we have taken a perturbation to the long-time mean profile

$$
\zeta=\bar{\zeta}^{\infty}(y)+\hat{\zeta}(y, t) e^{i m \theta}+\cdots
$$

For this study $m=2$, although $m$ is left arbitrary in the following equations for mathematical clarity. Solving for $\hat{\zeta}(y, t)$ as in $\S 3.2$, with the initial condition $\hat{\zeta}\left(y, 0^{+}\right)=0$, and substituting this into the equation for $\hat{\varphi}$ gives

$$
\partial_{t} \hat{\varphi}=-\frac{m \beta \pi}{2} \hat{\varphi}-\int_{0}^{t} K(t-s) \hat{\varphi}(s) d s,
$$

where the kernel

$$
K(t)=m \int_{-\infty}^{\infty} \partial_{y} \bar{\zeta}^{\infty} e^{-i m y t} d y
$$

Seeking a solution to (3.13) of the form $\hat{\varphi} \propto e^{\gamma t}$ leads to an implicit equation for the growth rate $\gamma$ :

$$
\gamma=-\frac{m \beta \pi}{2}-\int_{0}^{\infty} e^{-\gamma u} K(u) d u
$$

Substituting (3.8) into (3.14), integrating the kernel $K(t)$ in (3.15) by parts and then using residue calculations, we can express the equation for $\gamma$ in the implicit form

$$
\gamma=-\frac{m \pi}{2}-\frac{32 m^{3} \rho}{(\pi m+2 \gamma)^{3}}
$$

where $\beta$ has been set equal to unity, and

$$
\rho=i \pi \Gamma \Delta_{r}-\left(\Delta_{r}^{2}+\Delta_{i}^{2}-\frac{\pi^{2}}{4} \Gamma^{2}\right)
$$

is a constant which depends on the initial conditions.

For an arbitrary set of initial conditions, equation (3.16) can be solved to give a complex growth rate $\gamma=\gamma_{r}+i \gamma_{i}$. We are interested in neutrally stable solutions of this equation $\left(\gamma_{r}=0\right)$ which will correspond to the initial condition producing persistent nonlinear satellites. This is achieved by solving the quartic equation (3.16) for $\gamma$ with some initial set of parameters $\left(\Gamma, \Delta_{r}, \Delta_{i}\right)$ and singling out the growth rate with the smallest real part. With two of these parameters fixed, the third is varied until $\gamma_{r}=0$ whereupon a second parameter is then changed and the first is again updated until $\gamma_{r}=0$. With the third parameter held fixed, this maps out a curve in the two-dimensional plane which satisfies this constraint of neutral stability. For illustration we consider again the case when $\Delta_{i}=0$ and compare the neutral stability contour for this diagnostic (contour 2), with the contour given by (3.10) for the inflexion point diagnostic (contour 1); these contours are plotted in figure 2 .

Figure 2 shows that the neutral mode diagnostic (contour 2) produces a closed contour in the $(\Gamma, \Delta)$ plane, which has a much smaller region of decaying solutions than the inflection point diagnostic (contour 1). Both diagnostics agree at the points $\Gamma=0$, $\Delta= \pm \pi^{2} / 4 \sqrt{2} \simeq \pm 1.744$, which are given in BLSY. We expect the actual threshold contour of the fully nonlinear problem to differ from the neutral mode contour, because the nonlinear effects not present in the neutral mode analysis are important for the formation of satellites. 


\section{Numerical simulation and the first bounce diagnostic}

The two weakly nonlinear diagnostics formulated in $\S 3$ depend only on the form of the large time vorticity perturbation $\bar{\zeta}^{\infty}(y)$. The inflection point diagnostic is quite crude, and although the neutral mode diagnostic ensures a persistent perturbation, it is only based on a weakly nonlinear development, and so discards the higher order Fourier modes and their interactions. In this section we formulate a nonlinear quantitative diagnostic which comes from the numerical solution of (2.13-2.15). Equation (2.13) is solved by expanding $\zeta^{\prime}$ as a series of even Fourier harmonics:

$$
\zeta^{\prime}(y, \theta, t)=\sum_{n=-N}^{N} \zeta_{2 n}^{\prime}(y, t) e^{2 i n \theta} .
$$

To aid the numerical stability of our code, we introduce the diffusive term $R_{\text {eff }}^{-1} \partial_{y}^{2} \zeta^{\prime}$ to the right hand side of (2.13) (Hall et al. 2003b). The inclusion of this viscous term destroys any fine scale structure in the vorticity field, but this does not affect the threshold values we calculate. Moreover, we have checked that for $R_{\text {eff }} \geq 10^{5}$ the results are insensitive to the value of $R_{\text {eff }}$ : we set $R_{\text {eff }}=10^{5}$ and our results are, for practical purposes, inviscid. This system of equations is then marched forward in time using a Crank-Nicholson scheme for the advective and diffusive terms, and an Adam-Bashforth method for the nonlinear terms. The coordinate $y$ across the critical layer is treated via a finite difference scheme.

For simplicity the $\operatorname{PDE}(2.13)$ is discretized in the finite domain $y \in[-L, L]$; thus boundary conditions are imposed on $\zeta_{2 n}^{\prime}$ at $y= \pm L$ for $n=0,1, \ldots, N$. This is achieved by considering the large $-y$ asymptotic form of the solutions for each $\zeta_{2 n}^{\prime}$. It is possible to calculate numerous terms in these asymptotic expansions, however for numerical accuracy it is sufficient to use just the leading order terms. The large- $y$ conditions imposed are

$$
\begin{aligned}
\zeta_{0}^{\prime} & \sim 2 i t y^{-1}\left(\Gamma \hat{\varphi}^{*} e^{-2 i y t}-\Gamma^{*} \hat{\varphi} e^{2 i y t}\right), \\
\zeta_{2}^{\prime} & \sim-\beta \hat{\varphi} y^{-1}+\beta(\Delta-i \pi \Gamma / 2) y^{-1} e^{-2 i y t}, \\
\zeta_{4}^{\prime} & \sim 2 i t \Gamma \hat{\varphi} y^{-1} e^{-2 i y t}, \\
\zeta_{2 n}^{\prime} & \sim 0, \quad \forall n>2 .
\end{aligned}
$$

The numerical solution of $\hat{\varphi}$ contains oscillations which are due to the integral of $\left\langle\zeta_{2}^{\prime}\right\rangle$ in (2.14) being approximated over a finite range of $y$. These oscillations are removed at leading order by using (4.3) to approximate the integral in (2.14) outside the $y \in[-L, L]$ domain; this calculation is given in appendix A. This method however does not remove all the oscillations, which persist at the next order, and so we time average our solution to produce a smooth result, as is also described in appendix A.

In the present study we found it sufficient to set the parameter $L=20$. For all the threshold calculations in this paper we found using $M=5001$ grid points in the $y$ direction and $N=32$ equations to be the minimum values needed to give consistent results. For nonlinear calculations (i.e. larger $\Gamma$ and $\Delta$ ) just above the threshold for satellite formation these should be increased to $M=8001$ and $N=128$.

Figure 3 shows two results of the numerical solution of (2.13-2.15) when the initial condition parameters $\Gamma$ and $\Delta$ are both real. The correction to the mean azimuthal profile $\bar{\zeta}(y)$ is plotted at $t=40$ and compared with the $\bar{\zeta}^{\infty}(y)$ result in (3.8) for (a) $(\Gamma, \Delta)=(0,0.3)$ and $(\mathrm{b})(\Gamma, \Delta)=(0.3,0.3)$. For both cases the numerical solution (solid line) and the asymptotic model (dotted line) are in very good agreement with one another showing that the numerical code works correctly. The small difference between the two methods is due to higher order terms of the small amplitude expansion, and this difference 
(a)

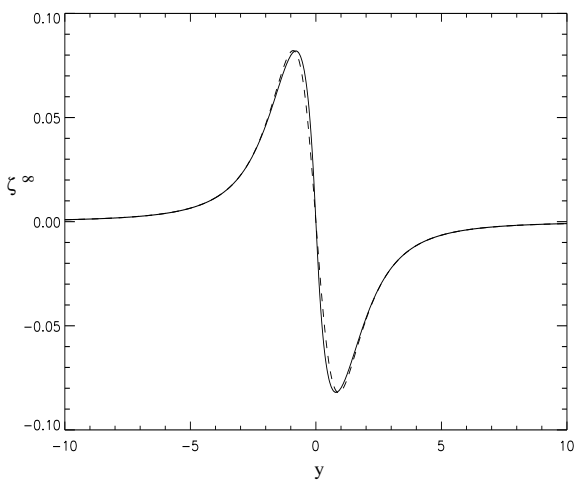

Figure 3. Figures of $\bar{\zeta}(y, t=40)$ (solid lines) and $\bar{\zeta}^{\infty}(y)$ (dotted lines) given by (3.8) for (a) $(\Gamma, \Delta)=(0,0.3)$ and $(\mathrm{b})(\Gamma, \Delta)=(0.3,0.3)$.

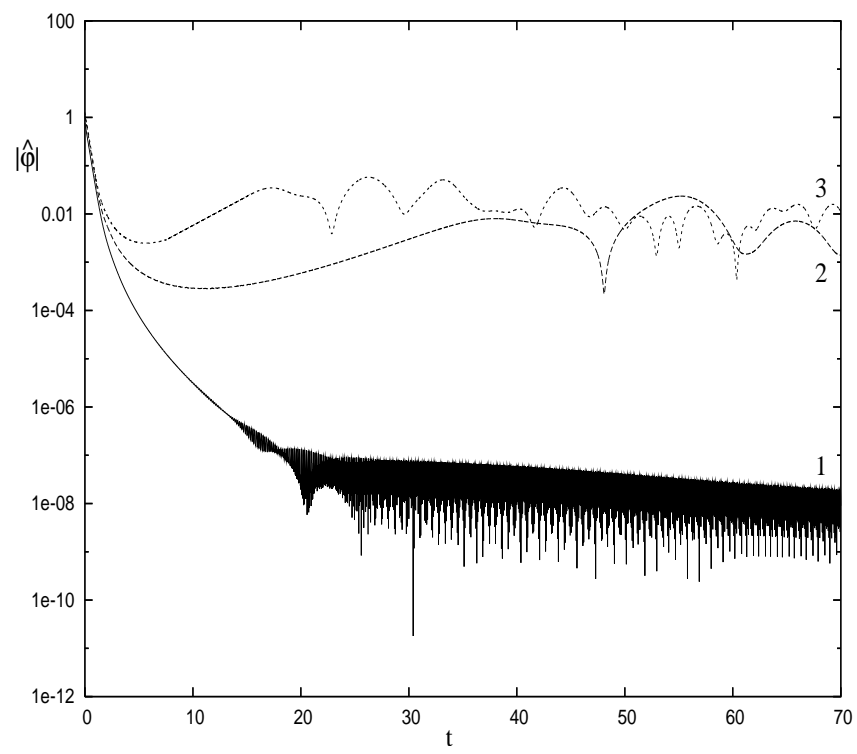

Figure 4. Plot of $|\hat{\varphi}(t)|$ for three typical numerical solutions of $(2.13-2.15)$ with $(\Gamma, \Delta)=(0.2,0.7)$ for curve $1,(\Gamma, \Delta)=(0.4,0.9)$ for curve 2 and $(\Gamma, \Delta)=(0.4,1.1)$ for curve 3.

increases as the parameters $\Gamma$ and $\Delta$ are increased towards the threshold values and beyond.

Figures 4 and 5 show typical results from the numerical solution of $(2.13-2.15)$. Figure 4 shows $|\hat{\varphi}|$ on a $\log$ scale as a function of $t$ for three runs with $(\Gamma, \Delta)=(0.2,0.7)$ for curve $1,(\Gamma, \Delta)=(0.4,0.9)$ for curve 2 and $(\Gamma, \Delta)=(0.4,1.1)$ for curve 3 . The parameter values for curve 1 correspond to a case where $|\hat{\varphi}|$ decays to very small values very quickly, and never increases again at large times. This is a weak initial condition run, where the vortex would return to an axisymmetric state and the perturbation would wind up. Curve 3 in figure 4 is a run with a strong initial condition for which $|\hat{\varphi}|$ rises in value after an initial period of decay and then oscillates about $|\hat{\varphi}|=0.01$. This corresponds to a case where satellites form in the critical layer, as can be seen in the total vorticity plot in figure 5(b). In this plot we can see the total vorticity $\zeta_{T}=y+\zeta$ winding around inside the satellites, and that due to the introduction of a non-zero $\Gamma$, these satellites are no longer situated 

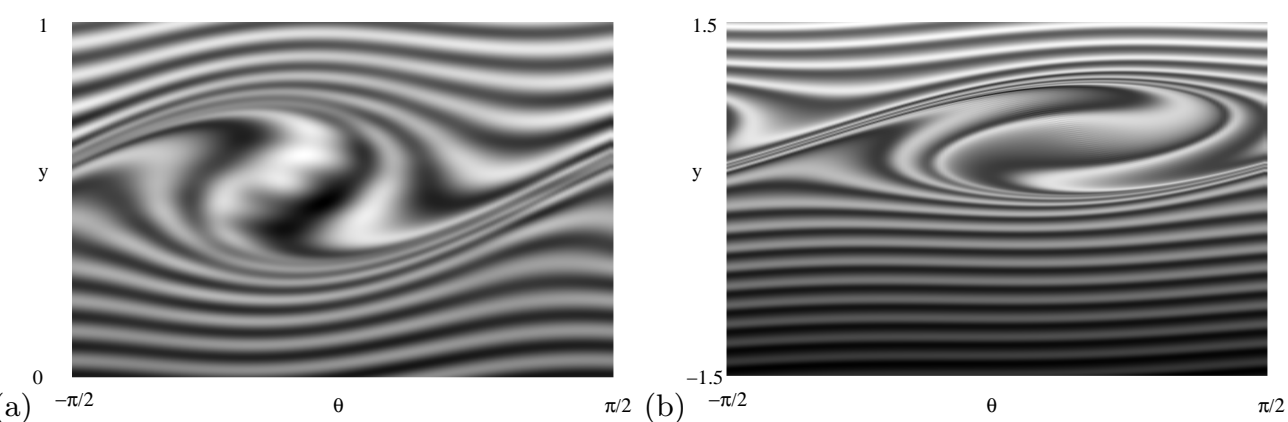

FiguRE 5. Grey-scale pictures of the total vorticity $\zeta_{T}=y+\zeta$ for $(\mathrm{a})(\Gamma, \Delta)=(0.4,0.9)$ and (b) $(\Gamma, \Delta)=(0.4,1.1)$. These snapshots are taken at $t=60$ in (a) and $t=40 \mathrm{in}(\mathrm{b})$ where positive vorticity is white and negative vorticity is black. Note that the vertical scale is different in each figure, and that the whole $y$ domain is not displayed.

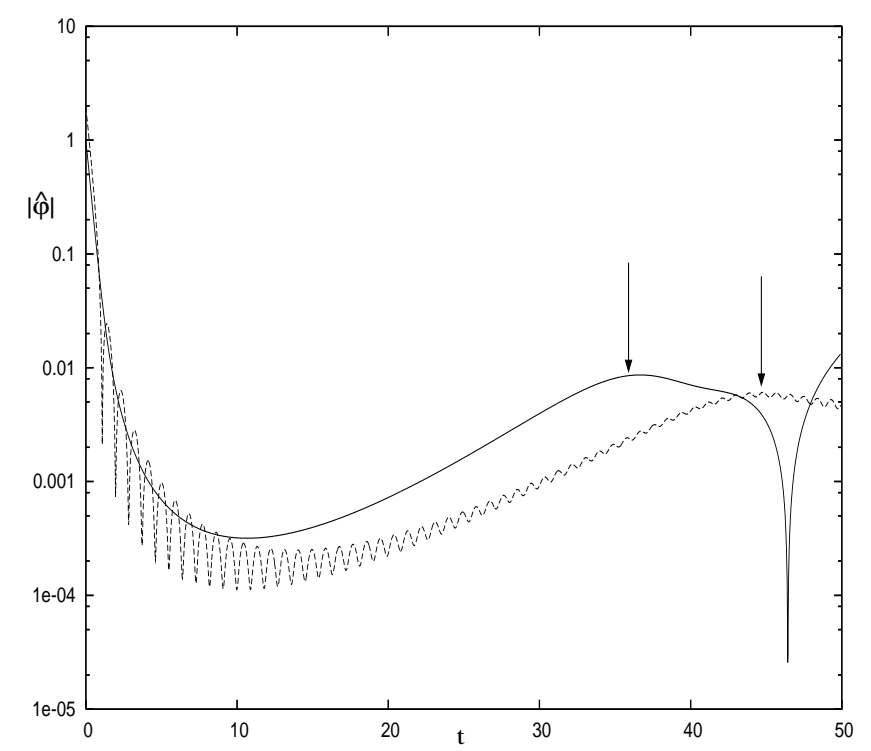

Figure 6. Plot of $|\hat{\varphi}(t)|$ on a log scale for $(\Gamma, \Delta)=(0,1)$ (solid line) and $(\Gamma, \Delta)=(1.25,0.05)$ (dashed line). The arrows denote the first bounce as defined in BLSY.

at $y=0$ as they would be if $\Gamma=0$ (see figure 16 of BLSY). Curve 2 in figure 4 represents a case between the other two, where $|\hat{\varphi}|$ does rise after an initial period of decay, but it does not oscillate around quite as rapidly as curve 3 . The total vorticity for curve 2 at $t=60$ can be seen in figure $5(\mathrm{a})$. This plot again shows the vorticity winding around in the critical layer, but in this case the winding is not so clear, and also there is some breaking up of the flow in the centre of the satellite. We shall see later that this case lies on the threshold between persistent satellites and decaying solutions. These three parameter values are plotted as the stars in figure 2 in $\S 3$.

The study of BLSY determines whether or not generated cat's eyes persist or decay away by considering the 'first bounce' in the stream function, $|\hat{\varphi}(t)|$. The first bounce is the position of the first maximum in the stream function that occurs after the initial exponential decay. The position of the first bounce is depicted by the arrows in figure 6. When $\Delta \gtrsim 0.1,|\hat{\varphi}(t)|$ is a smooth function (see the solid line in figure 6 ), and so measuring the position of the first bounce is simple. However when $\Delta$ is small and $\Gamma$ is 
moderately large (see dashed line in figure 6), then there is a feed back from the vorticity in the critical layer to the stream function and vice versa. Thus $|\hat{\varphi}(t)|$ contains oscillations and so calculating the position of the first bounce is more complicated. For this case a first bounce fit is done by eye rather than calculating the position of this turning point in the time-averaged stream function.

In their study, BLSY use as a criterion that if the first bounce occurs after $t=45$ then cat's eyes do not persist. This time value places an upper bound on the threshold contour and avoids numerical error which can accumulate in long time integrations with small values of $\hat{\varphi}$. As we find that our results are insensitive to the precise choice of this cut-off time, we adopt this same criterion for our study. To calculate the threshold curve for this diagnostic with $\Gamma$ and $\Delta$ real, we reduce $\Delta$ for a fixed $\Gamma$ until the first bounce occurs after $t=45$. This will generate a contour in the $(\Gamma, \Delta)$ plane within which satellites decay and outside which they persist.

The first bounce threshold described above is plotted as contour 3 in figure 2 along with the inflection point threshold (contour 1) and the neutral mode threshold (contour 2 ). We see that the first bounce threshold gives a much smaller region of the parameter space where satellites decay, although the shape of this contour is very similar to that of the neutral mode threshold and the difference between the two is only roughly a factor of 2 . The point on the contour where $\Gamma=0(\Delta \simeq 0.97)$ agrees exactly with the value for an instantaneous kick $(T=0)$ in figure 13 of BLSY. Although increasing the cut-off time $t=45$ used in our criterion will bring the contour in slightly, the numerical evidence from simulations of $(2.13-2.15)$ is that the effect is very minor, the position of the contour is insensitive to this choice and that for disturbances inside this contour the stream function ultimately decays. In the next section we examine this statement further by running full nonlinear simulations of the Navier-Stokes equations and comparing the results to the threshold given by the asymptotic model.

\section{Comparison with Navier-Stokes simulations}

In $\S 4$ we calculated the first bounce threshold in parameter space using the asymptotic theory of BLSY, within which satellites decay and the vortex returns to an axisymmetric state and outside which they persist and the vortex remains elliptical. In this section we form a link from the parameter values in this asymptotic method to the parameter values related to full Navier-Stokes simulations in (1.1), (1.3) and (1.4). Our simulations will show that there is a region of parameter space where the asymptotic model agrees well with the full numerical simulations, and outside this region, where the agreement is not so good, we offer explanations as to why this is the case. We fix $m=2$ in what follows, with the critical layer at $r_{2}=\sqrt{2}$.

In the full simulations we solve the Navier-Stokes equations using a numerical scheme which incorporates 128 even Fourier modes in the azimuthal $\theta$ direction and a finite difference method consisting of 1500 points in the radial $r$ direction. The code integrates the time derivative and the diffusive term together using a Crank-Nicholson scheme, while the nonlinear terms are incorporated explicitly via the Adam-Bashforth method. For more information on this numerical scheme see Turner \& Gilbert (2007). The initial condition used in the simulations is given by (1.1) where $Z_{0}(r)$ is as in (1.3), but here we write

$$
Z_{2}(r)=\frac{r^{a}}{4 \pi} \frac{1-\tanh \left(\left(r^{2}-\sigma_{2}^{2}\right) /\left(4\left(1-\sigma_{2}\right)\right)\right)}{1+\tanh \left(\sigma_{2}^{2} /\left(4\left(1-\sigma_{2}\right)\right)\right)}
$$




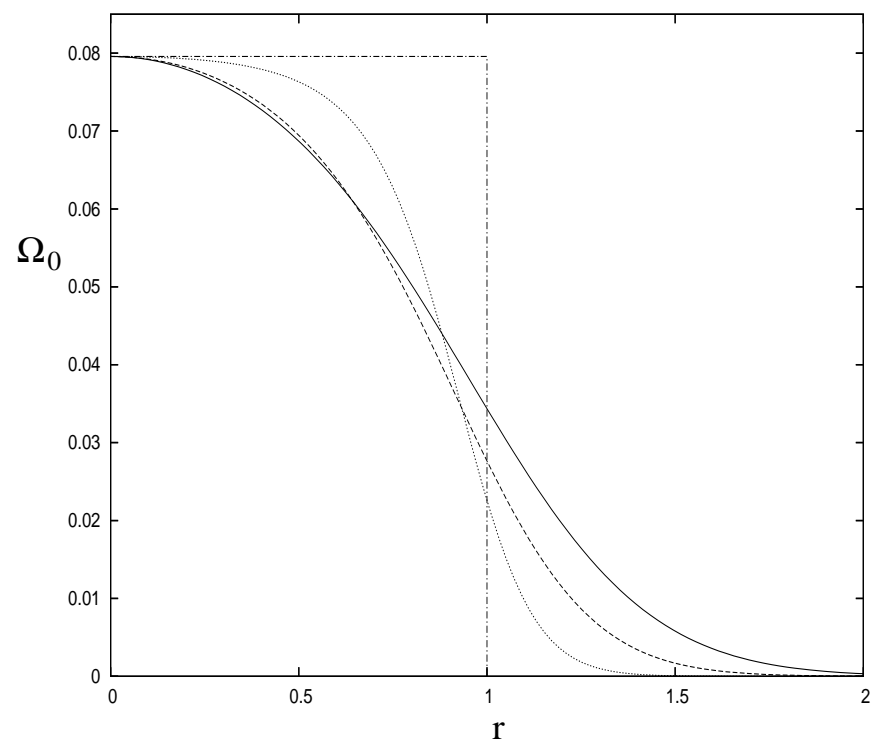

Figure 7. Plot of the vorticity profile (1.3) for $\sigma=0.7,0.8,0.9$ and 1 represented by the solid, dashed, dotted and dot dashed lines respectively.

where

$$
a=\frac{1}{2\left(1-\sigma_{2}\right)}\left(1+\tanh \left(\frac{1+\sigma_{2}}{4}\right)\right) .
$$

The reason for this is because now the maximum value of $Z_{2}(r)$ occurs at $r=1$ for all $\sigma_{2}$, which improves the comparison with the asymptotic theory as the position of the maximum of $Z_{2}(r)$ now agrees with that of $g(r)$ in (B 2). However, this form of $Z_{2}(r)$ is only appropriate for $\sigma_{2}>0.65$ because then $a>2$ and the function $Z_{2}(r)$ is relatively smooth at the origin.

The parameter $\sigma$ in (1.3) is chosen close to 1 so that the vortex resembles a Rankine vortex plus a small asymptotic skirt $\epsilon Z_{S}(r)$ as in (1.5). Thus when calculating $\epsilon Z_{S}$ at the critical layer $r=r_{2}$, outside the core of the vortex, we identify this with $Z_{0}\left(r_{2}\right)$. Figure 7 plots the vorticity profile (1.3) with $\sigma=0.7,0.8,0.9$ and 1 showing that as $\sigma$ approaches 1 the profile tends to a Rankine vortex. The two parameters $\delta$ and $\sigma_{2}$ in (1.3) and (5.1) are linked to the asymptotic parameters $\Gamma$ and $\Delta$ via (2.7). For a fixed value of $\sigma_{2}$, varying $\delta$ moves us along a straight line in the $(\Gamma, \Delta)$ plane, while changing $\sigma_{2}$ varies the angle between this straight line and the line $\Gamma=0$.

For the numerical simulations presented here, we set $\sigma_{2}=\sigma$ and vary $\sigma$. For each $\sigma$ we ran our numerical simulation for various values of $\delta$ and by considering the magnitude of the second multipole moment $Q_{2}(t)$ where, in general,

$$
Q_{m}(t)=\int_{0}^{\infty} r^{m+1} \zeta_{m} d r
$$

we were able to determine if satellites persist, signaled by $\left|Q_{2}(t)\right|$ not decaying. By way of illustration, figure 8 plots $\ln \left|Q_{2}(t)\right|$ for $\sigma=\sigma_{2}=0.78$ and $\delta=0.037$ (solid line) and $\delta=0.111$ (dotted line). The solid line is a case where $\left|Q_{2}\right|$ decays and the perturbation decays, while the dotted line is a case where $\left|Q_{2}\right|$ oscillates and the satellites persist. All of the numerical simulations were run with a Reynolds number $R=10^{7}$, which at first sight appears large enough to ensure that the results are inviscid for practical 


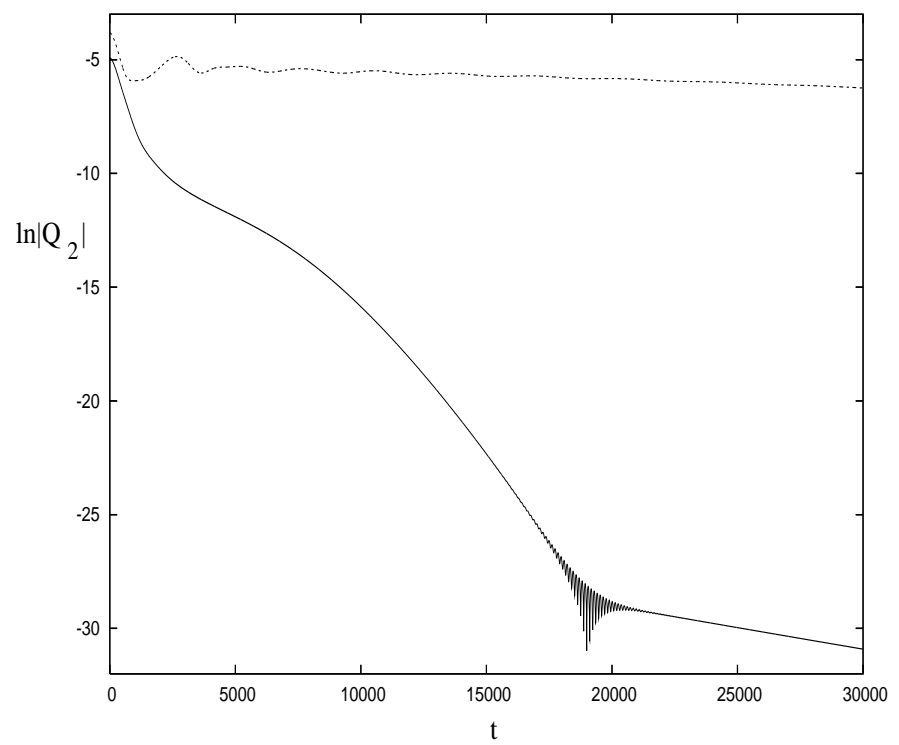

Figure 8. Plot of $\left|Q_{2}(t)\right|$ for $\sigma=\sigma_{2}=0.78$ and $\delta=0.037$ (solid line) and $\delta=0.111$ (dotted line). The solid line is a case where the satellites decay and the vortex becomes axisymmetric, and the dotted line is one where the satellites persist.

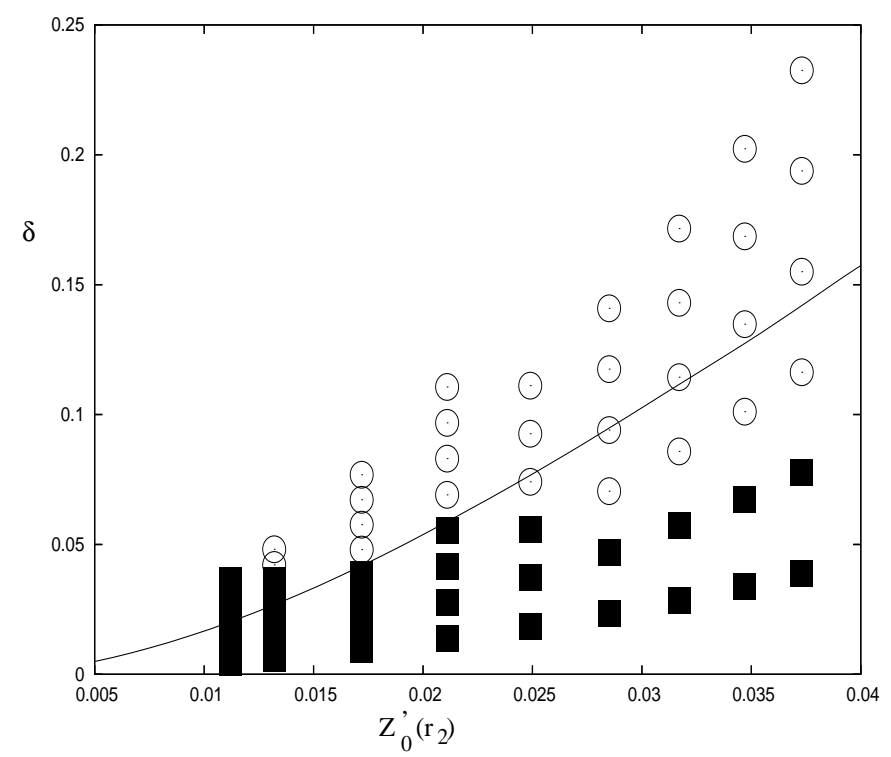

Figure 9. Plot of $\delta$ as a function of $Z_{0}^{\prime}\left(r_{2}\right)$. The circles depict simulations where $\left|Q_{2}(t)\right|$ persists, and the squares where it decays. The solid line represents contour 3 in figure 2, inside which the nonlinear perturbation decays.

purposes. However as we will see shortly this Reynolds number maps to the effective Reynolds number, $R_{\text {eff }}$, for the asymptotic theory which in certain parameter ranges may no longer be large enough to guarantee that the evolution is practically inviscid on the (short) spatial and (long) temporal scales of the critical layer.

Figure 9 plots $Z_{0}^{\prime}\left(r_{2}\right)$ against $\delta$, where our full Navier-Stokes simulations are repre- 


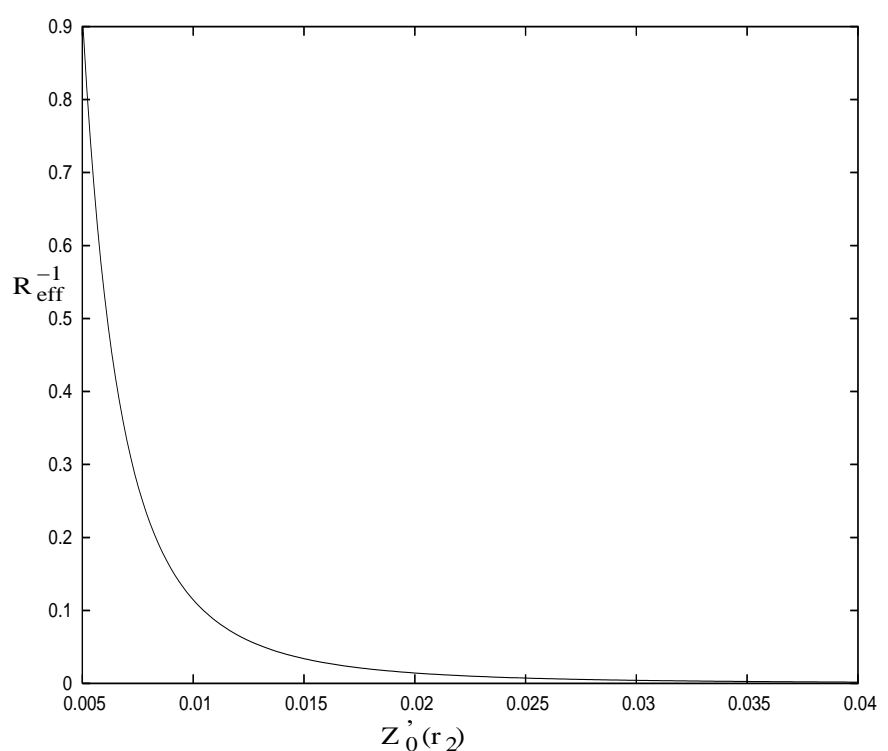

Figure 10. Plot of the effective diffusivity, $R_{\mathrm{eff}}^{-1}$ as a function of $Z_{0}^{\prime}\left(r_{2}\right)$ for $R=10^{7}$.

sented by points in this plane. The solid line indicates the asymptotic result given by contour 3 in figure 2 , and $Z_{0}^{\prime}\left(r_{2}\right) \equiv \epsilon Z_{S}^{\prime}\left(r_{2}\right)$ is the small parameter from the BLSY theory. The squares in figure 9 represent runs where the amplitude of the multipole moment $Q_{2}(t)$ decays sufficiently that the vortex enters a regime that is governed by the core of the vortex (Bassom \& Gilbert 1998) (see solid line in figure 8) and hence would not then grow at a later time. The circles represent runs where the amplitude of the multipole moment has not started to decay after a sufficiently long run (up to $t=5 \times 10^{4}$ ). We see that the agreement between the numerical simulations and the asymptotic theory improves as the small parameter $Z_{0}^{\prime}\left(r_{2}\right)$ decreases, which is as expected because this value is equivalent to the small parameter $\epsilon$ in the asymptotic theory. Note that the range of good agreement occurs for $Z_{0}^{\prime}\left(r_{2}\right) \leq 0.021(\sigma \geq 0.8)$; by examining the vorticity profiles in figure 7 we can see that the $\sigma=0.8$ profile is actually quite broad and thus this asymptotic theory agrees well with the numerical simulations for reasonably broad vortices as well ones with sharper edges. However, for $Z_{0}^{\prime}\left(r_{2}\right) \leq 0.0132$ (or $\sigma \geq 0.84$ ) we find that the good agreement begins to evaporate: at parameter values where satellites should persist according to the asymptotic model, the full simulation shows decay. This can be explained by considering the Reynolds number $R$ from the full numerical simulations and the effective Reynolds number of the asymptotic theory $R_{\text {eff }}$ based on the spatial and temporal scales in the critical layer; these both conspire to make $R_{\text {eff }}$ much less than $R$ for small $\epsilon$. More specifically, these two quantities are related via the expression

$$
R_{\text {eff }}^{-1}=R^{-1} \epsilon^{-3} \mathcal{L}^{-2} \mathcal{T}
$$

where $\mathcal{L}$ and $\mathcal{T}$ are the length and time scales given in (B 7) of appendix B (Hall et al. $2003 b$ ). When the limiting compact vortex is the Rankine vortex, as for our simulations, we obtain

$$
R_{\mathrm{eff}}^{-1}=\frac{8 \sqrt{2} R^{-1}}{\pi^{2}\left|Z_{0}^{\prime}\left(r_{2}\right)\right|^{3}} .
$$

The effective Reynolds number for the case when $R=10^{7}$ is plotted in figure 10 . For the values of $0.016<Z_{0}^{\prime}\left(r_{2}\right)<0.021$ we obtain good agreement between the numerical 

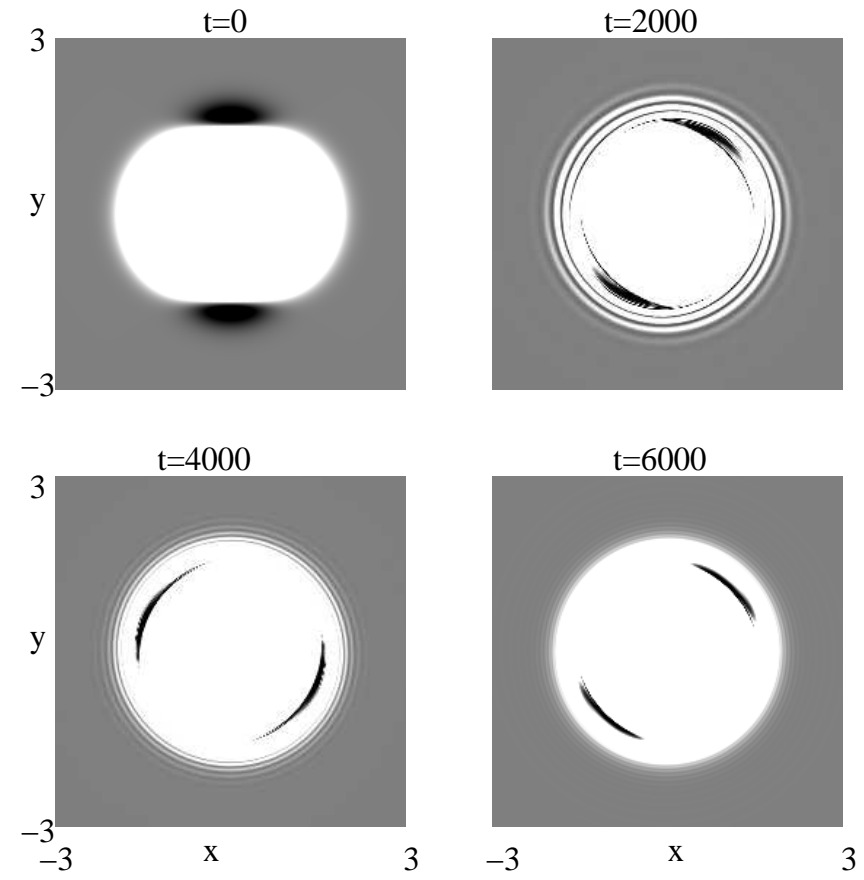

FIGURE 11. Plot of the vorticity $Z$ showing persistent negative satellites for $\sigma=\sigma_{2}=0.8$ and $\delta=0.2212$. The vorticity is capped at $|Z|=0.0001$ (white), zero vorticity appears as gray and negative vorticity is black.

simulations and the asymptotic theory (see figure 9) and we see from figure 10 that the effective diffusivity $R_{\text {eff }}^{-1}$ is reasonably small in this region. Thus any diffusive effects due to the critical layer being very thin are small, and the results are the same as the asymptotic theory in $\S 4$, where we fixed $R_{\text {eff }}=10^{5}$. For $Z_{0}^{\prime}\left(r_{2}\right)>0.021, R_{\text {eff }}^{-1}$ decreases further and so the reason for the disagreement between the results in figure 9 in this region is not due to diffusive effects, but is simply that our vorticity profile $Z_{0}(r)$ is no longer close enough to the asymptotic limit of Rankine vortex plus a skirt of vanishing strength.

However, as we decrease $Z_{0}^{\prime}\left(r_{2}\right)$ from 0.016 our vorticity profile becomes much more like a Rankine vortex plus a skirt, so we expect our full simulations to agree better with the asymptotic theory. Unfortunately because the critical layer becomes thinner, the effective Reynolds number decreases and diffusive effects become more significant. This problem can be overcome by increasing the Reynolds number $R$ of the full simulation. When a simulation plotted in figure 9 shows persisting satellites, we observe that increasing $R$ does not make them decay, but it can make a decaying simulation grow or persist by making the problem more inviscid. Results of the full simulation with $R=10^{8}, 10^{9}$ and $10^{10}$ show that the decay observed above the solid line in figure 9 for $Z_{0}^{\prime}\left(r_{2}\right)<0.016$ appears to turn into persistence and growth, while the parameter values below the line still give decay. However, at these large Reynolds numbers the full numerical simulation could not be run for the same length of time as the $R=10^{7}$ case; although we could not verify that these results would persist to the same accuracy as in figure 9 , the evidence we have does suggest this is the case. Overall, we believe that our numerical results indicate that the asymptotic theory is correct, and discrepancies for very sharp vortices are down to the limits of running simulations at sufficiently high Reynolds numbers. 
In the asymptotic theory the sign of vorticity in the critical layer is of secondary consideration as the constant $\Gamma_{0}$ in (2.5) has no influence on the dynamics. Thus when satellites are formed they could equally well be positive, cat's eyes or negative satellites giving a tripole, depending on the value of $\Gamma_{0}$. We note that a similar issue arises in the Navier-Stokes simulations, as a constant can be added to the initial vorticity profile, corresponding to working in a rotating frame. This has led us to conclude that both a tripole and a vortex with cat's eyes amount to the same nonlinear structure, but the sign of the vorticity in the satellites is entirely dependent on the average level of vorticity around the critical layer initially.

We highlight this below with a typical Navier-Stokes simulation. The form of the initial vorticity profile (1.1) is that of a positive elliptical core with two negative regions of vorticity above and below the core (with $m=2$ ), this can be seen in the $t=0$ panel of figure 11. Figure 11 shows the evolution of an initial vorticity distribution with $\sigma_{2}=\sigma=0.8$ and $\delta=0.2212$. This case is one which has persistent satellites. The vorticity plotted is capped at $|Z|=10^{-4}$. The positive vorticity is white in the figure, grey signifies zero vorticity and the black regions are regions of negative vorticity. We see in figure 11 that the satellites do persist for long times, and the vorticity in the critical layer includes negative inclusions (depicted as black). This means that this is a tripole with a positive core and two negative satellites rotating around it as in figure 1. However, adding on the constant $Z_{\min }$ to the initial profile (1.1), where $Z_{\min }$ is the minimum vorticity in the run depicted in figure 11, would produce a vortex with the same structure as in figure 11 except the black regions would now correspond to regions of positive vorticity. Note that we could add on a constant globally, or just locally within the critical layer by modifying the initial profile.

The simulation with $\delta=0.2212$ in figure 11 is well above the threshold for persistent satellites, as can be seen in figure 9. For simulations just above the threshold for persistence, the satellites are very thin and viscous effects come into play: in a plot similar to figure 11 it would be difficult to see the sign of the vorticity in the critical layer, and so whether there are positive or negative satellites present.

\section{Conclusions}

In this paper we have studied the evolution of a sharp edged vortex with an additive perturbation via both asymptotic and numerical methods. We adapt the asymptotic theory of BLSY so that we can stipulate an initial amplitude of mode $m$ vorticity in the critical layer and an initial stream function amplitude of the perturbation. In the case when these two parameters are real we were able to construct a contour in this parameter space, within which the stream function decays in time and the vortex returns to an axisymmetric state and outside which the vortex remains elliptical and a satellite structure persists in the vortex. The results from this paper were all constructed with $Z_{2}(r)$ real, and hence $\Gamma$ and $\Delta$ real, to agree with the studies of Rossi et al. (1997) and Barba \& Leonard (2007), but could be extended to cover the full three-dimensional parameter space. Also it should be noted that these results are universal in that they apply to any family of vortices for which the BLSY theory becomes valid, in other words which asymptotically have the structure of a compact vortex supporting a normal mode, plus a weak skirt of vorticity. Thus by considering the family of compact vortices in BLSY one could calculate $\Gamma$ and $\Delta$, via (B 10) and (B 13), for the Gaussian profiles used by Rossi et al. (1997) and Barba \& Leonard (2007), and hence qualitatively verify their results.

We compared our first bounce threshold from the nonlinear study to two thresholds 
based on weakly nonlinear theory, an inflection point threshold and a neutral mode threshold. The inflection point threshold was very crude and did not agree well with the first bounce threshold, however the neutral mode threshold agreed in shape with the first bounce threshold and was only out by a factor of two in magnitude.

The first bounce threshold was compared to full numerical simulations of the NavierStokes equations. These simulations showed that as our core vortex approached a Rankine vortex plus an asymptotic skirt the numerical results agree well with the asymptotic results while the effective Reynolds number was large. The Navier-Stokes simulations, like the BLSY model, are unaffected by the addition of a constant vorticity initially. This has led us to conclude that these satellite structures successfully describe both multipole and cat's eye vortices, i.e these two types of vortices are essentially the same, except their azimuthal averaged profiles will be different (Barba \& Leonard 2007; Turner \& Gilbert 2007). In the context of geophysical applications and two-dimensional turbulence, sharp edged vortices are common place and our study indicates that the thresholds for the formulation of tripole structures will tend to be low in cases when the critical layer lies beyond the sharp edge. Of course continual external forcing of such vortices is likely to lead generally to a more complex vorticity structure in the layer, and a 'surf zone' can occur (Thuburn \& Lagneau 1999). The enhanced mixing that occurs in such zones can in tern sharpen up the edge of the vortex, and this interaction of mixing and profile evolution is currently under investigation in random and deterministic contexts.

\section{Acknowledgments}

This work was undertaken on the EPSRC grant EP/D032202/1. We would also like to thank Lorena Barba, Andrew Bassom and John Thuburn for useful comments in the preparation of this paper. AG was recipient of a Leverhulme Trust Research Fellowship during this study.

\section{Appendix A. Asymptotic approximation of integral the in (2.14) outside of $y \in[-L, L]$}

In this appendix we show how the large- $y$ asymptotic solutions to (2.13-2.15) are used to approximate the contribution to the integral $\left\langle\zeta_{2}^{\prime}\right\rangle$ in (2.14) from outside the domain $y \in[-L, L]$. To calculate the correction terms to this integral outside this domain up to $O\left(L^{-1}\right)$, we require the $O\left(y^{-2}\right)$ terms in (4.3). These can be easily calculated and give

$$
\zeta_{2}^{\prime} \sim-\frac{\beta \hat{\varphi}}{y}+\frac{\beta(\Delta-i \pi \Gamma / 2) e^{-2 i y t}}{y}-\frac{i \beta \hat{\varphi}_{t}}{2 y^{2}}-\frac{2 t^{2} \hat{\varphi}^{2} \Gamma^{*} e^{2 i y t}}{y^{2}}-\frac{i \beta^{2} \pi}{2 y^{2}}\left(\Delta-\frac{i \pi \Gamma}{2}\right) e^{-2 i y t} .
$$

When substituted into (2.14) this leads to

$$
\begin{aligned}
\left\langle\zeta_{2}^{\prime}\right\rangle & =\left\langle\zeta_{2}^{\prime}\right\rangle_{L}-\frac{i}{L} \hat{\varphi}_{t}-2 i\left(1-\beta^{2} \pi t\right)\left(\Delta-\frac{i \pi \Gamma}{2}\right) \int_{L}^{\infty} \frac{\sin (2 y t)}{y} d y \\
& -\beta^{2} \pi\left(\Delta-\frac{i \pi \Gamma}{2}\right) \frac{\cos (2 L t)}{L}+O\left(L^{-2}\right)
\end{aligned}
$$

where \langle\rangle$_{L}$ is the principal value integral evaluated between $y=-L$ and $L$. This approach is equivalent to that in appendix A of BLSY, however in this case the non-zero initial conditions produce a more complicated expression.

The inclusion of these correction terms in (2.14) eliminates the oscillations that occur in the numerical solution of $\hat{\varphi}$ at leading order. However oscillations remain at the 


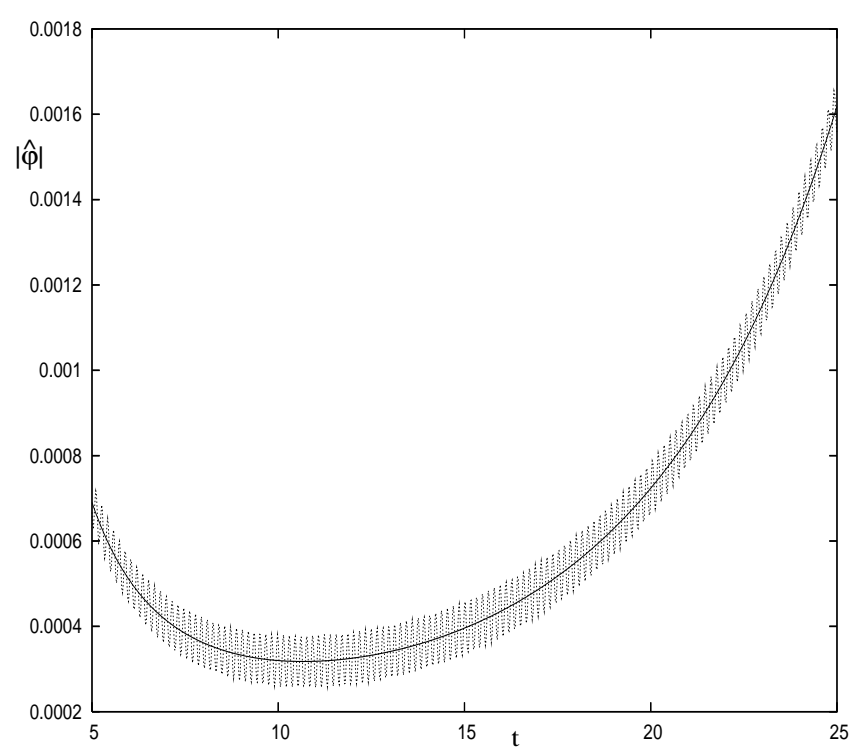

Figure 12. Plot of $|\hat{\varphi}(t)|$ for $(\Gamma, \Delta)=(0,1)$. The dotted line shows the results before time averaging and the solid line after time averaging.

order of $L^{-2}$, and we have not tried to eliminate these completely because we would require many correction terms to do this. Thus, to produce a smooth function of $\hat{\varphi}(t)$, for plotting and calculation purposes, we time average the solution over the period $\pi / L$ of the oscillations. As an example, plots of $|\hat{\varphi}(t)|$ with $(\Gamma, \Delta)=(0,1)$ with and without time-averaging are shown in figure 12 . As $L$ is increased, the period of the oscillations reduces as $O\left(L^{-2}\right)$, as does the amplitude at the same rate; however it is not practical to remove the oscillations this way as this requires an increase in the number of mesh points in the $y$ direction which increases the running time of the code making it numerically impractical. We found that varying $L$ produces consistent time-averaged results.

\section{Appendix B. Asymptotic theory}

In this appendix we give a brief overview of quantities from the derivation of the asymptotic theory used in the paper in particular (B 10), (B 13) and (B 24). The reader is directed to BLSY for more detailed information. We seek an asymptotic solution for the total vorticity $Z$ of the Navier-Stokes equation in the form

$$
Z(r)=Z_{C}(r)+\epsilon Z_{S}(r)+\epsilon^{2} \zeta^{0}+\epsilon^{3} \zeta^{1}+\cdots .
$$

The quantity $Z_{C}(r)$ is the vorticity profile of a compact vortex, which we take to be a Rankine vortex in the main paper, $Z_{S}(r)$ is an asymptotic skirt and $\epsilon \ll 1$. In the main body of the paper the quantity $Z_{C}(r)+\epsilon Z_{S}(r)$ is combined into the full profile $Z_{0}(r)$, which is the tanh profile for our full simulations.

We consider perturbations to the compact vortex: such a vortex may support a family of (undamped) Kelvin waves, at most one for each mode $m$. Each Kelvin wave has a critical radius $r_{m}$, where the vortex and the fluid co-rotate with angular frequency $\omega_{m}$. We let the functions $f(r)$ and $g(r)$ be the corresponding linear perturbations to the 
stream function and vorticity. For the Rankine vortex these are

$$
f_{m}(r)=\left\{\begin{array}{ll}
\left(r_{m} r\right)^{m}, & (r<1), \\
\left(r_{m} / r\right)^{m}, & (r>1),
\end{array} \quad g_{m}(r)=-2 m r_{m}^{m} \delta(r-1) .\right.
$$

Outside the critical layer at $r=r_{m}$, the solution is the Kelvin wave with a complex amplitude that evolves, slowly, according to the behaviour in the critical layer,

$$
\left[\psi^{0}, \zeta^{0}\right]=a(\vartheta, \tau)[f(r), g(r)] .
$$

Here $\vartheta=\theta-\omega_{m} t, \tau=\epsilon t$ is a slow timescale and $a(\vartheta, \tau)=\hat{a}(\tau) e^{i m \vartheta}+$ c.c. is the amplitude function, where $r_{m}=(m /(m-1))^{1 / 2}$. In deriving $(2.1-2.4)$ the following quantities appear

$$
\mathcal{I}_{1}=-\int_{0}^{R_{C}} \frac{r f g}{m r_{m} \widetilde{\Omega}_{C}} d r, \quad \widetilde{\Omega}_{C} \equiv \Omega_{C}(r)-\Omega_{C}\left(r_{m}\right),
$$

where $R_{C}$ is the radius of the compact vortex, beyond which $Z_{C}=0$.

The gradient of the vorticity inside the critical layer scales as

$$
\beta \equiv \operatorname{sgn}\left(\mu_{m}\right)=-\operatorname{sgn}\left(Z_{S}^{\prime}\left(r_{m}\right)\right),
$$

where

$$
\mu_{m} \equiv \frac{Z_{S}^{\prime}\left(r_{m}\right)}{r_{m} \widetilde{\Omega}_{C}^{\prime}\left(r_{m}\right)} .
$$

When $\beta=-1$ the vortex has a positive skirt and hence gives unstable solutions. As we are only interested in stable solutions we set $\beta=1$ in this study. The system has a second rescaling by introducing the space and time scales

$$
\mathcal{L} \equiv-\frac{\left|\mu_{m}\right|}{\mathcal{I}_{1} \widetilde{\Omega}_{C}^{\prime}\left(r_{m}\right)}, \quad \mathcal{T} \equiv \frac{\mathcal{I}_{1}}{\left|\mu_{m}\right|} .
$$

To determine the constants $\Gamma$ and $\Delta$ in terms of $\delta$ and $Z_{m}(r)$, consider the initial vorticity distribution of the fully nonlinear Navier-Stokes simulations

$$
Z=Z_{C}(r)+\epsilon Z_{S}(r)+\delta Z_{m}(r) e^{i m \theta}+\text { c.c., }
$$

where $\delta$ and the form of the mode $Z_{m}(r)$ determine the values of $\Gamma$ and $\Delta$. In this appendix we show how these parameters are related to the parameter $\delta$ of the fully nonlinear problem. In the critical layer at $t=0$ we compare the expansion (B 8) with

$$
Z=Z_{C}(r)+\epsilon Z_{S}(r)+\epsilon^{2} \zeta^{0}(r, \theta)+\epsilon^{3} \zeta^{1}(r, \theta)+\cdots
$$

Omitting some details on the scalings (to be found in BLSY), when this is rescaled we find the amplitude of the mode $m$ in the critical layer to be

$$
\Gamma=\frac{\mathcal{T}}{r_{m}\left|\mu_{m}\right|} \epsilon^{-2} \delta Z_{m}\left(r_{m}\right)=\frac{r_{m} \mathcal{I}_{1} \widetilde{\Omega}_{C}^{\prime 2}\left(r_{m}\right)}{\epsilon^{2} Z_{S}^{\prime 2}\left(r_{m}\right)} \delta Z_{m}\left(r_{m}\right)
$$

Outside the critical layer we compare (B 8) at $t=0$ with the expansion

$$
Z=Z_{C}(r)+\epsilon Z_{S}(r)+\epsilon^{2} \hat{a}(0) g(r) e^{i m \theta}+\text { c.c. }+\cdots
$$

where the function $g(r)$ for a Rankine vortex is defined in (B 2). At leading order we can neglect the effect of the skirt and just consider perturbations to the compact vortex $Z=Z_{C}$. The initial perturbation $\delta Z_{m}(r) e^{i m \theta}$ will excite the normal mode by an amount we call $\delta P_{m}$ and it will also excite the continuous spectrum. The latter perturbations will wind up and become finely scaled on the fast $t$-timescale, and hence are irrelevant. 
However the perturbation to the normal mode will evolve on the slow $\tau$-timescale and so will be significant.

It is sufficient to write the initial condition as

$$
\hat{a}(0)=\epsilon^{-2} \delta P_{m}
$$

where $P_{m}$ is the projection onto the normal mode, to be determined shortly. Rescaling this initial condition we find

$$
\Delta=\hat{\varphi}(0)=\frac{\mathcal{T}}{r_{m} \mathcal{L}} \hat{a}(0)=-\frac{r_{m} \mathcal{I}_{1}^{2} \widetilde{\Omega}_{C}^{\prime 3}\left(r_{m}\right)}{\epsilon^{2} Z_{S}^{\prime 2}\left(r_{m}\right)} \delta P_{m}
$$

To find the value of the projection $P_{m}$ we consider the linearized form of the NavierStokes equation by writing

$$
\zeta=\zeta_{0}(r)+\zeta_{m}(r, t) e^{i m \theta} \quad \text { and } \quad \psi=\psi_{0}(r)+\psi_{m}(r, t) e^{i m \theta} .
$$

We then take the Laplace transform of the resulting equation with respect to $t$, with

$$
\bar{f}_{m}(p)=\int_{0}^{\infty} e^{i p t} f_{m}(t) d t, \quad f_{m}(t)=-\frac{1}{2 \pi} \int_{\infty+i \gamma}^{-\infty+i \gamma} e^{-i p t} \bar{f}_{m}(p) d p .
$$

It can be shown that the Laplace transform of the multipole moment $Q_{m}(t)$ in (5.3) can be written as

$$
\bar{Q}_{m}(p)=\lim _{r_{0} \rightarrow \infty}-\frac{i r_{0}^{m}}{\Psi_{L}\left(r_{0}, p\right)} \int_{0}^{r_{0}} \frac{s \Psi_{L}(s, p) \zeta_{m}(s, 0)}{m \Omega_{C}(s)-p} d s,
$$

(Schecter et al. 2000; Turner \& Gilbert 2007), where $\Psi_{L}(r, p)$ satisfies the homogeneous equation

$$
\left[\frac{\partial^{2}}{\partial r^{2}}+\frac{1}{r} \frac{\partial}{\partial r}-\frac{m^{2}}{r^{2}}+\frac{m Z_{C}^{\prime}(r)}{r\left(p-m \Omega_{C}(r)\right)}\right] \Psi_{L}(r, p)=0,
$$

with $\Psi_{L}(0, p)=0$. Here $r=r_{0}$ is a large radius, that is taken to be finite and then allowed to tend to infinity. When (B 16) is inverted to find $Q_{m}(t)$, the inversion contour is closed around any singularities in the $p$-plane. These singularities can occur because of the normal mode, when $\Psi_{L}\left(r_{0} \rightarrow \infty, p\right)$ vanishes for some $p$, or because $m \Omega_{C}(s)-p$ vanishes for some $s$ in the range 0 to $\infty$. The second of these is the continuous spectrum of the vortex, which may be bent below the real $p$-axis by moving the $s$-contour above the real $s$-axis in (B 16) (Briggs et al. 1970; Schecter et al. 2000).

Examining (B 17) shows that this is exactly the equation satisfied by the functions $f(r)$ and $g(r)$ and thus the solution for the normal mode is $p=m \omega_{m}$ and $\Psi_{L}(r, p)=f(r, p)$. Now inverting (B 16) by deforming the contour around the singularity at $p=m \omega_{m}$ only, we find that

$$
Q_{m}(t)=-i R \int_{0}^{\infty} \frac{s f\left(s, m \omega_{m}\right) \zeta_{m}(s, 0)}{m\left(\Omega_{C}(r)-\omega_{m}\right)} d s e^{-i m \omega_{m} t}
$$

where $R$ is the residue at $p=m \omega_{m}$,

$$
R=\underset{p=m \omega_{m}}{\operatorname{Res}}\left[\lim _{r_{0} \rightarrow \infty}\left(-\frac{i r_{0}^{m}}{f\left(r_{0}, p\right)}\right)\right] .
$$

To calculate $P_{m}$ from this expression, we calculate $Q_{m}(0)$ for our initial condition $Z_{m}(r)$ and we divide by $Q_{m}^{N M}(0)$ for the normal mode, where $\zeta_{m}(r, 0)$ is replaced by $g(r)$ and the corresponding projection $P_{m}=1$ by definition. We find that

$$
Q_{m}^{N M}(0)=i R r_{m} \mathcal{I}_{1},
$$


and thus

$$
P_{m}=-\mathcal{I}_{1}^{-1} \int_{0}^{\infty} \frac{s f(s) Z_{m}(s)}{m r_{m} \widetilde{\Omega}_{C}(s)} d s,
$$

where the contour of integration is bent above the singularity at $r_{m}$ given by $\widetilde{\Omega}_{C}\left(r_{m}\right)=0$. As we have bent the contour above the pole at $r_{m}$ we can write the expression for $P_{m}$ as a principal value integral plus a contribution from the pole,

$$
P_{m}=-\mathcal{I}_{1}^{-1}\left(\mathrm{PV} \int_{0}^{\infty} \frac{s f(s) Z_{m}(s)}{m r_{m} \widetilde{\Omega}_{C}(s)} d s-\frac{i \pi}{m} \frac{Z_{m}\left(r_{m}\right)}{\widetilde{\Omega}_{C}^{\prime}\left(r_{m}\right)}\right) .
$$

However we realise that the imaginary part here looks familiar: on its own, when inserted into $\Delta$ in (B 13) it gives a term

$$
-\frac{i \pi}{m} \frac{r_{m} \mathcal{I}_{1} \widetilde{\Omega}_{C}^{\prime 2}\left(r_{m}\right)}{\epsilon^{2} Z_{S}^{\prime 2}\left(r_{m}\right)} \delta Z_{m}\left(r_{m}\right) .
$$

For $m=2$, this is precisely $-i \pi / 2$ times the quantity $\Gamma$ and what we are recovering is the initial delta-function forcing of the quasi-mode amplitude $\Delta$ by the vorticity in the critical layer, a quantity we disentangled in (2.15). This is a useful check, but to avoid accounting for this twice we delete the imaginary part of $P_{m}$ to leave as our definition

$$
P_{m}=-\mathcal{I}_{1}^{-1} \mathrm{PV} \int_{0}^{\infty} \frac{s f(s) Z_{m}(s)}{m r_{m} \widetilde{\Omega}_{C}(s)} d r .
$$

which in the case of the Rankine vortex amounts to (2.8).

\section{REFERENCES}

Bajer, K., Bassom, A. P. \& Gilbert, A. D. 2001 Accelerated diffusion in the centre of a vortex. J. Fluid Mech. 437, 395-411.

Balmforth, N. J., Llewellyn Smith, S. G. \& Young, W. R. 2001 Disturbing vortices. J. Fluid Mech. 426, 95-133 (referred to herein as BLSY).

BArba, L. A. 2006 Self-organization of vortex multipoles at high Reynolds number. Phys. Rev. E 73, 065303(R).

Barba, L. A. \& LeOnard, A. 2007 Emergence and evolution of tripole vortices from netcirculation initial conditions. Physics of Fluids 19 (1), 017101.

Bassom, A. P. \& Gilbert, A. D. 1998 The spiral wind-up of vorticity in an inviscid planar vortex. J. Fluid Mech. 371, 109-140.

Bernoff, A. J. \& Lingevitch, J. F. 1994 Rapid relaxation of an axisymmetric vortex. Phys. Fluids 6, 3717-3723.

Briggs, R. J., Daugherty, J. D. \& Levy, R. H. 1970 Role of Landau damping in crossed-field electron beams and inviscid shear flow. Phys. Fluids 13, 421-432.

Carton, X. \& Legras, B. 1994 The life-cycle of tripoles in two-dimensional incompressible flows. J. Fluid Mech. 267, 53-82.

Dritschel, D. G. 1989 On the stabilization of a two-dimensional vortex strip by adverse shear. J. Fluid Mech. 206, 193-221.

Dritschel, D. G. 1998 On the persistence of non-axisymmetric vortices in inviscid twodimensional flows. J. Fluid Mech. 371, 141-155.

Fornberg, B. 1977 A numerical study of 2-D turbulence. J. Comput. Phys. 25, 1-31.

Hall, I. M., Bassom, A. P. \& Gilbert, A. D. 2003a The effect of fine structure on the stability of planar vortices. Eur. J. Mech. B Fluids 22 (2), 179-198.

Hall, I. M., Bassom, A. P. \& Gilbert, A. D. $2003 b$ The effect of viscosity on the stability of planar vortices with fine structure. Quart. J. Mech. Appl. Math. 56 (4), 649-657.

Koumoutsakos, P. 1997 Inviscid axisymmetrization of an elliptical vortex. J. Comput. Phys. $138(2), 821-857$. 
LE Dizès, S. 2000 Non-axisymmetric vortices in two-dimensional flows. J. Fluid Mech. 406, $175-198$.

Legras, B. \& Dritschel, D. 1993 Vortex stripping and the generation of high vorticity gradients in two-dimensional flows. Appl. Sci. Res. 51, 445-455.

Llewellyn Smith, S. G. 1995 The influence of circulation on the stability of vortices to modeone disturbances. Proc. R. Soc. Lond. A 451, 747-755.

Macaskill, C., Bassom, A. P. \& Gilbert, A. D. 2002 Nonlinear wind-up in a strained planar vortex. Eur. J. Mech. B Fluids 21 (3), 293-306.

MCWilliams, J. C. 1984 The emergence of isolated coherent vortices in turbulent flow. J. Fluid Mech. 146, 21-43.

Melander, M. V., McWilliams, J. C. \& Zabusky, N. J. 1987 Axisymmetrization and vorticity-gradient intensification of an isolated two-dimensional vortex through filamentation. J. Fluid Mech. 178, 137-159.

Möller, J. D. \& Montgomery, M. T. 1999 Vortex Rossby waves and hurricane intensification in a barotropic model. J. Atmospheric Sci. 56 (11), 1674-1687.

Morel, Y. G. \& CARTON, X. J. 1994 Multipolar vortices in two-dimensional incompressible flows. J. Fluid Mech. 267, 23-51.

Pingree, R. D. \& Le Cann, B. 1992 Anticyclonic eddy X91 in the southern bay of Biscay, May 1991 to February 1992. J. Geophys. Res. 97, 14353-14367.

Rossi, L. F., Lingevitch, J. F. \& Bernoff, A. J. 1997 Quasi-steady monopole and tripole attractors for relaxing vortices. Phys. Fluids 9 (8), 2329-2338.

Schecter, D. A., Dubin, D. H. E., Cass, A. C., Driscoll, C. F., Lansky, I. M. \& O’Neil, T. M. 2000 Inviscid damping of asymmetries on a two-dimensional vortex. Phys. Fluids 12 (10), 2397-2412.

Thuburn, J. \& Lagneau, V. 1999 Eulerian mean, contour integral, and finite-amplitude wave activity diagnostics applied to a single-layer model of the winter Stratosphere. J. Atmospheric Sci. 56 (5), 689-710.

Turner, M. R. \& Gilbert, A. D. 2007 Linear and nonlinear decay of cat's eyes in twodimensional vortices, and the link to Landau poles. J. Fluid Mech. 593, 255-279.

Turner, M. R., Gilbert, A. D. \& BAssom, A. P. 2008 Neutral modes of a two-dimensional vortex and their link to persistent cat's eyes. Phys. Fluids 20 (2), 027101-1-027101-10.

Van Heisst, G. J. F., Kloosterziel, R. C. \& Williams, C. W. M. 1991 Laboratory experiments on the tripolar vortex in a rotating fluid. J. Fluid Mech. 225, 301-331. 